\title{
ANTHROPEN
}

Le dictionnaire francophone d'anthropologie ancré dans le contemporain

\section{CHOC CULTUREL}

Genest, Sylvie, Université du Québec à Montréal, Canada

Gouin-Bonenfant, Mathilde, Université de Cambridge, Angleterre

White, Bob, Université de Montréal, Canada

Date de publication : 2021-12-08

DOI: https://doi.org/10.47854/anthropen.v1i1.41078

Voir d'autres entrées dans le dictionnaire

En anthropologie, l'expression choc culture/ désigne à la fois l'expérience forte du dépaysement vécu par les personnes qui se trouvent immergées dans un environnement culturel étranger et le concept heuristique utilisé en recherche universitaire pour classer les données collectées dans le champ des études en communication interculturelle.

La différence entre expérience et concept est importante. Alors que l'expérience présente une grande variété de formes et d'intensités, le concept tel qu'il s'est répandu dans l'imaginaire populaire en restreint l'observation à certains aspects seulement, se concentrant sur les expériences d'où naissent des émotions négatives et des comportements jugés inhabituels. Or, pour les anthropologues, toutes les manifestations (au niveau individuel mais aussi au niveau des collectivités) présentent un intérêt dans la mesure où elles sont considérées comme des indicateurs de la mise en marche d'un processus d'adaptation des personnes qui se trouvent dans un contexte social ou culturel qui leur est étranger, autrement dit à l'échelle d'un système.

Du point de vue de la recherche, le processus d'adaptation individuel, qui est plutôt personnel et circonstanciel, diffère du processus d'acculturation, qui est davantage social et structurel. Le premier est aussi plus circonscrit dans le temps : en croisant les données de la durée et de la teneur des états des personnes en processus d'adaptation culturelle, des chercheurs ont établi des courbes (en « $U$ » ou en "W 》) illustrant le tout sous forme graphique. Les analyses ont conduit à l'identification de trois à cinq étapes d'adaptation qui peuvent s'étaler sur une 
période de 6 à 24 mois (Lysgaard 1955 ; Gullahorn et Gullahorn 1963). Au terme de ce processus, l'adaptation des voyageurs à leur nouvel environnement culturel est supposée complète. Dans les cas les plus graves de dépaysement, les personnes affectées semblent apaisées par des séjours plus ou moins longs et fréquents dans des espaces contrôlés agissant comme «tampon culturel », de même que par la perspective de leur rapatriement.

Le choc culturel est abordé par la psychologie et certaines sciences appliquées comme un état passager réunissant un ensemble plus ou moins stable de symptômes liés à un "syndrome général d'adaptation " ou à une "anxiété du dépaysement ». Dans ce cas, il est étudié à travers différentes théories du domaine de la santé (principalement celles du stress) et selon différentes approches qui se distinguent par une polarisation, soit autour des événements critiques (approche situationniste), soit autour des dispositions ou des compétences des personnes (approche personnaliste), soit autour de l'interaction entre les individus et leur environnement (approche interactionniste). Ces différentes approches brossent du choc culturel un «tableau clinique » très variable qui se présente comme un " état de malaise [ayant] des effets différents, des degrés de gravité différents et des durées différentes selon les personnes " (Barna 1994 : 344). Des études plus récentes et plus ciblées arrivent néanmoins à mettre en évidence des ensembles assez stables de signes et de comportements, de sorte à rendre possible la catégorisation des affections du voyageur sous la forme de syndromes spécifiques.

En sciences sociales, le concept de choc culturel est profondément enraciné dans I'histoire de l'anthropologie américaine du début du XXe siècle. Sa logique repose principalement sur les idées soutenues dans les années 1920 à 1950 par l'École " culture et personnalité » dont les principes ont été exposés par les étudiants de Boas (Sapir, Benedict, Linton, Herskowitz, Mead, Du Bois). Dans ce cadre théorique, le concept de choc culturel est sous-tendu par une conception des cultures, pensées au pluriel et conçues comme étant spécifiques, homogènes et dotées d'une " configuration » leur donnant une cohérence interne (patterns of culture) (Benedict 1934). L'élaboration d'un discours thérapeutique autour du concept et de l'expérience du choc culturel par les anthropologues manifeste en outre les rapports entre l'anthropologie culturelle et la psychiatrie établis par les tenants de l'École culturaliste américaine entre 1920 et 1950.

Dutton (2012) a déjà fait état des difficultés à identifier la source originale et la première utilisation de l'expression « choc culturel » (culture shock, cultural shock) en sciences sociales. En anthropologie, des témoignages laissent penser que le terme était utilisé par boutade et sans prétention scientifique par les étudiants de Boas pour désigner la «maladie professionnelle » des anthropologues sur leur terrain de recherche au cours des années 1930 (Du Bois 1951). L'expression semble par ailleurs avoir été « bien connue dans le cas de l'immigrant » dans les années précédant la publication de l'ouvrage de Carpenter (1931:272). Gamio 
utilise par exemple l'expression cultural shock pour signaler une différence de forme et de fond entre la "civilisation » des immigrants mexicains et celle du peuple américain (Gamio 1929). L'expression est aussi mobilisée dans la sociologie urbaine américaine des années 1920-1950, par le biais de l'expérience de l'« urban-rural conflict » (Park et Burgess 1921 ; Carpenter 1931 ; Holt 1940) : ce dernier usage rappelle les travaux de l'école de Chicago sur la désorganisation sociale en faisant référence à des troubles psychologiques ou à la criminalité. II apparaît enfin qu'aux États-Unis, le terme fut « si largement utilisé » par le milieu savant qu'il ne nécessitait plus « aucune citation » (Seymour 2015 : 372, note 31). Si le démenti qu'oppose Dutton à la paternité d'Oberg quant à la création de l'expression « choc culturel » est clair, complet et définitif, il garde toutefois sous silence le plagiat que paraît aussi avoir commis Oberg (1960) en paraphrasant ou même en intégrant à son texte des phrases complètes de Cora Du Bois (1951).

À partir des années 1950, l'idée que toute personne «transplantée » en milieu étranger puisse être perturbée par une forme de désadaptation culturelle a stimulé la prolifération de programmes de formation subventionnés visant à préparer les voyageurs américains (diplomates, soldats, volontaires ou professionnels en missions humanitaires, commerciales ou autres) aux conséquences potentielles du choc culturel. Dans la perspective d'une contribution des anthropologues à la sécurité nationale et à la promotion de la démocratie à travers le monde, notamment au cours de la guerre froide, ces programmes ont été développés et dispensés à travers des structures telles que le Foreign Service Institute (1947), le People-to-People program - Travel That Changes You (1956) et le programme Peace Corps Volunteers (1960). Parmi les solutions envisagées par les anthropologues dans ces cadres de formation, Oberg et Hall (1957) ont tous deux pensé « qu'un bon moyen d'atténuer le choc culturel était d'en informer les gens à l'avance » (Calhoun $1977: 125)$.

Les écrits mettent en évidence deux cadres interprétatifs du choc culturel. Un premier cadre met en valeur une conception mécaniste du mot choc, ce qui lui donne le sens métaphorique de "collision ». Ce cadre prend sa source dans le contexte de la révolution moderne technologique, artistique et urbaine, largement commentée par des auteurs comme Edgar Alan Poe, Charles Baudelaire et Georg Simmel. Vu leur intérêt littéraire et leur pouvoir d'évocation, les métaphores physicalistes ont pris une place relativement importante dans le discours universitaire sur les cultures au début du XXe siècle.

Un deuxième cadre interprétatif permet d'appréhender le choc culturel comme une psychopathologie se classant, selon les auteurs ou les époques, le long d'un spectre allant de l'anxiété sévère au trouble d'adaptation en passant par « des manifestations à la fois physiques et psychologiques [incluant] des nausées, une désorientation, une réaction hostile envers les nouvelles personnes et les nouveaux lieux, et qui s'accompagne parfois d'un désir aigu de rentrer chez soi immédiatement » (Smith 2008 : 41). En s'établissant sur des données empiriques, 
les études de ce type dépassent le niveau des approches intuitives pour faire du concept de choc culturel un instrument de détection des processus culturels ou psycho-pathologiques. Dans ce contexte, le concept de choc culturel permet d'évaluer des signes et des comportements, d'identifier des symptômes et de classer des syndromes en fonction de leurs manifestations.

Certaines critiques font toutefois valoir que l'introduction, dans les théories du changement, d'une conception du choc comme «épreuve culturelle » réduit la complexité des phénomènes anthropologiques : d'une part, la conception du choc en termes de trouble laisse dans la marge une partie des données, notamment celles qui documentent les cas où "l'expérience de l'étranger [...] offre des possibilités qui sont précieuses pour la croissance personnelle et intellectuelle » (Meintel $1973: 55$ ) ; d'autre part, une telle conception ne tient compte que des processus individuels en négligeant ce qui se passe à "l'intersection de deux cultures » (Berry $2005:$ 708).

Des critiques d'un autre ordre ont des conséquences encore plus dommageables sur l'utilité du concept de choc culturel dans le cadre de la réflexion anthropologique. On lui reproche de n'être associé " à aucune théorie culturelle ou psychologique ni à aucun contexte de recherche " (Berry 2005 : 708) en plus de le soupçonner d'admettre " certaines suppositions non scientifiques » (Dutton 2012). Néanmoins, dans sa version la plus aboutie, la recherche met de l'avant une démarche inductive, propre au raisonnement clinique et à l'enquête ethnographique, faisant du choc culturel un concept opératoire bénéficiant d'une large littérature destinée à la formation et à l'information des praticiens (Ward, Bochner et Furnham 2001). Or, si l'anthropologie a pris ses distances avec le concept de choc culturel depuis les années 1970 (Bennett 2013), on peut se demander si c'est par crainte d'essentialisme méthodologique ou si c'est plutôt parce que, dans sa tradition disciplinaire, l'anthropologie a eu davantage d'intérêt pour la complexité des groupes particuliers que pour l'analyse des interactions entre les groupes.

En situant le concept de choc culturel dans le contexte plus large des théories du changement, un troisième cadre interprétatif encore peu installé dans la recherche anthropologique semble offrir une autre option. Ce cadre donne une valeur actualisée au concept de choc culturel en lui substituant le concept plus neutre de seuil du changement systémique. Cette formulation s'intègre à une théorie des changements de type logique qui adviennent dans la cognition humaine lorsqu'une situation de communication paradoxale vient en dérégler les fonctions adaptatives (Bateson 1972). Cette voie du développement théorique fondée sur la pensée systémique de Bateson permet d'élaborer une explication qui ne présume pas de l'issue positive ou négative de l'expérience du choc, qui peut être utilisée à différentes échelles d'analyse (individuelle, groupale, humaine), qui transcende le vocabulaire spécialisé de la psychologie et qui demeure proche des préoccupations de l'anthropologie. 


\section{Références}

Barna, L.M. (1994), "Stumbling Blocks in Intercultural Communication », dans L.A. Samovar, R.E. Porter et E.R. McDaniel, Intercultural Communication: $A$ Reader, 7e édition. Belmont (CA), Wadsworth Publishing Company, p. 337346.

Bateson, G. (1972), Steps to an Ecology of Mind, San Francisco, Chandler Publishing Company.

Bennett, M. (2013), «The Ravages of Reification: Considering the Iceberg and Cultural Intelligence, Towards De-reifying Intercultural Competence », communication présentée lors de la conférence FILE IV, Colle Val d’Elsa (Italie) le 28 septembre.

Benedict, R. (1959 [1934]), Patterns of Culture, Boston, Houghton Mifflin.

Berry, J.W. (2005), "Acculturation: Living successfully in two cultures ", International Journal of Intercultural Relations, vol. 29, n 6 , p. 697-712. https://doi.org/10.1016/j.jijtrel.2005.07.013

Carpenter, N. (1931), The Sociology of City Life, New York, Longsman, Green and Company.

Calhoun, T.W. (1977), Culture shock and its effects on American teachers in overseas schools: an exploratory study, thèse de doctorat, ScholarWorks@UMass Amherst, Doctoral Dissertations 1896-February 2014, Amherst, University of Massachusetts.

https://scholarworks.umass.edu/cgi/viewcontent.cgi?article=4126\&context= dissertations_1

Du Bois, C. (1951), «Culture Shock », communication, Midwest Regional Meeting of the Institute of International Education, Chicago, 28 novembre.

Dutton, E. (2012), Culture Shock and Multiculturalism: Reclaiming a Useful Model from the Religious Realm, Newcastle upon Tyne (R.-U.), Cambridge Scholar Publishing.

Gamio, M. (1929), "Observations on Mexican Immigration Into the United States ", Pacific Affairs, vol. 2, nº 8, p. 463-469.

Gullahorn, J.T. et J.E. Gullahorn (1963), "An Extension of the U-Curve Hypothesis ", Journal of Social Issues, vol. 19, $\mathrm{n}^{\circ}$ 3, p. 33-47. https://doi.org/10.1111/j.1540-4560.1963.tb00447.x

Hall, E.T. et K. Oberg (1957), «Things to Know About Culture », Community Development Review, $\mathrm{n}^{\circ}$ 7, décembre, p. 1-4.

Holt, J.B. (1940), « Holiness Religion: Cultural Shock and Social Reorganization ", American Sociological Review, vol. 5, n 5, p. 740-747.

Lysgaard, S. (1955), "Adjustment in a Foreign Society: Norwegian Fulbright Grantees Visiting the United States », International Social Science Bulletin, $\mathrm{n}^{\circ} 7$, p. $45-51$.

Meintel, D. (1973), «Strangers, Homecomers and Ordinary Men », Anthropological Quarterly, vol. 46, n 1, numéro thématique « Ethnographic Fieldwork: A Mirror for Self and Culture », p. 47-58. 
Oberg, K. (1960), "Cultural shock: Adjustment to new cultural environments ", Missiology: An International Review, vol. 7, $\mathrm{n}^{\circ}$ 4, p. 177-182. https://journals.sagepub.com/doi/10.1177/009182966000700405

Park, R.E. et E.W. Burgess (1921), Introduction to the Science of Sociology, Chicago, University of Chicago Press.

Seymour, S. (2015), Cora Du Bois: Anthropologist, Diplomat, Agent, Lincoln et Londres, University of Nebraska Press.

Smith, D.C. (2008), "Pulling the Plug on Culture Shock: A Seven Step Plan for Managing Travel Anxiety », The Journal of Global Business Issues, vol. 2, $\mathrm{n}^{\circ} 1$.

Ward, C., S. Bochner et A. Furnham (2001), The Psychology of Culture Shock, Londres, Routledge. 